\title{
Transoral robotic surgery and neck dissection as primary treatment results in reduced adjuvant treatment for patients with advanced oropharyngeal squamous cell cancer
}

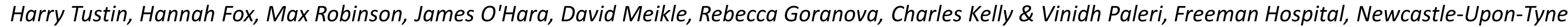
Objectives

To establish if primary treatment of advanced oropharyngeal squamous cell carcinoma (OPSCC) with transoral robotic surgery (TORS) and neck dissection leads to deintensification of adjuvant treatment relative to treatment of equivalent disease with primary chemoradiotherapy.

\section{Methods}

All patients who had TORS and neck dissection for advanced (stage III/IV) OPSCC between March 2013 and December 2016 were identified from our prospective dataset. Pathology results and adjuvant treatment regimes were obtained by retrospective review of clinical records.

Discussion with the local oncology team determined that treatment without surgery would have been primary chemoradiotherapy with a radiotherapy dose of 65Gy to the primary tumour and ipsilateral neck for all cases in the series. Patients with advanced nodal status $(\geq 2 \mathrm{~b})$ would also have had radiotherapy to the contralateral neck. Treatment de-escalation resulting from surgery was considered in terms of avoidance of chemotherapy, reduction in radiotherapy dosage and avoidance of contralateral neck radiotherapy.

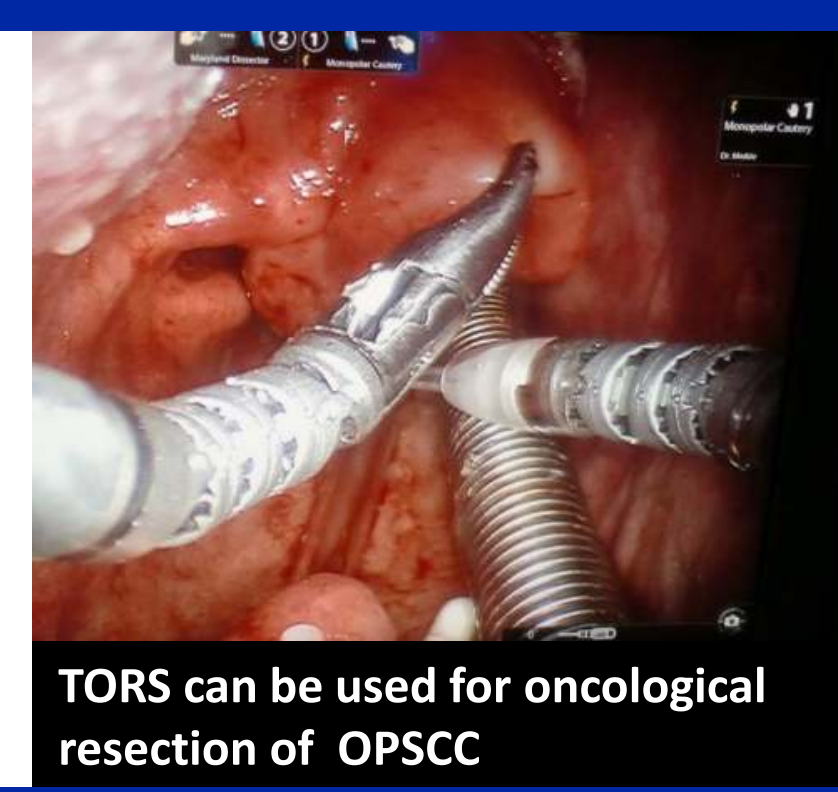

\section{Results}

32 patients with advanced OPSCC had TORS and neck dissection and received adjuvant treatment determined by institutional protocol.

\section{Requirement for adjuvant chemotherapy:}

In cases with \& without extranodal extension (ENE) of nodal metastases

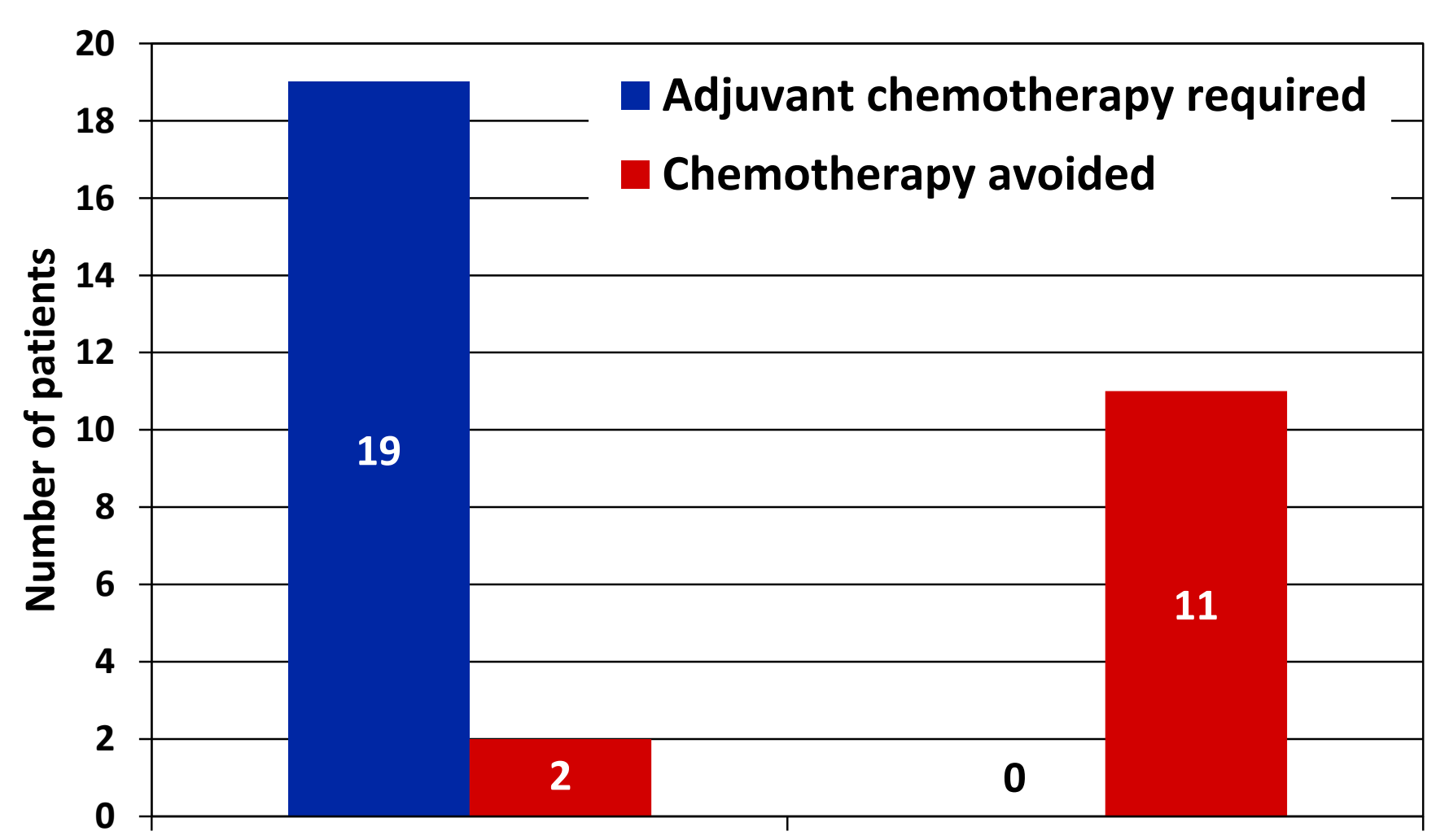

ENE of nodal metasteses

No ENE of nodal metasteses
Radiotherapy to the primary tumour bed

- Reduced dose possible in 21/32 (63\%)

- Mean adjuvant radiotherapy dose: 59.7Gy

Radiotherapy to the ipsilateral neck

- Reduced dose possible in 20/32 (63\%)

- Mean adjuvant radiotherapy dose: 61.6Gy

Radiotherapy to the contralateral neck

24 patients had N2b, N2c or N3 nodal disease. Without surgery all would have required contralateral neck radiotherapy

- $18(75 \%)$ avoided contralateral neck radiotherapy

- $6(25 \%)$ had radiotherapy to both sides of the neck

Summary of reductions to adjuvant treatment following TORS and neck dissection

\begin{tabular}{|l|c|c|c|c|c|c|c|c|c|c|}
\hline Percentage of patients & $\mathbf{2 5 \%}$ & $\mathbf{1 3 \%}$ & $\mathbf{3} \%$ & $\mathbf{1 3 \%}$ & $\mathbf{9 \%}$ & $\mathbf{9 \%}$ & $\mathbf{3 \%}$ & $\mathbf{3 \%}$ & $\mathbf{3} \%$ & $19 \%$ \\
\hline Chemotherapy avoided (Yes / No) & Yes & Yes & Yes & No & No & No & No & No & No & No \\
\hline $\begin{array}{l}\text { Reduced radiation dose to primary } \\
\text { tumour bed (Yes / No) }\end{array}$ & Yes & Yes & No & Yes & No & Yes & Yes & Yes & Yes & No \\
\hline $\begin{array}{l}\text { Reduced radiation dose to } \\
\text { ipsilateral neck (Yes / No) }\end{array}$ & Yes & Yes & Yes & Yes & No & Yes & No & No & No & No \\
\hline $\begin{array}{l}\text { Avoidance of contralateral neck } \\
\text { radiation (Yes / No / N/A (if nodal } \\
\text { stage <2b)) }\end{array}$ & Yes & N/A & N/A & Yes & Yes & N/A & Yes & Yes & No & No \\
\hline
\end{tabular}

**81\% experienced reduction to their treatment following surgery in terms of reduced radiotherapy \&/or avoidance of chemotherapy**

\section{Conclusions}

In patients with pathological stage III/IV (TNM7) OPSCC, TORS with neck dissection allowed reduction in radiotherapy dosage to the primary tumour bed and ipsilateral neck and in most cases with advanced nodal status, enabled avoidance of contralateral neck radiotherapy. In cases where there was no ENE of nodal metastases, patients were able to avoid chemotherapy as a result of this surgery 